\title{
Electron Tomographic Analysis of the Assembly of cis-Golgi cisternae and of Cell Plates
}

\author{
L. A. Staehelin, ${ }^{*}$ J. R. Austin,* B. S. Donohoe, ${ }^{*}$ Z. R. Gergely, ${ }^{*}$ B.-H. Kang, ${ }^{*}$ M. S. Otegui ${ }^{+}$and J. \\ M. Segui-Simarro* \\ * Department of Molecular, Cellular and Developmental Biology, UCB 347, University of Colorado, \\ Boulder, CO 80309 \\ ${ }^{+}$Department of Botany, University of Wisconsin, Madison, WI 53706
}

Cis-Golgi cisternae and plant cell plates are two membrane compartments that are created de novo from vesicles derived from other organelles of the secretory pathway. In particular, cis-Golgi cisternae are produced from COPII vesicles that bud from endoplasmic reticulum (ER) export sites, and the cell plates of dividing plant cells are assembled from vesicles derived from the trans Golgi Network (TGN). To characterize the membrane assembly intermediates involved in these processes, we have high pressure frozen and freeze-substituted plant and algal cells, and determined their 3D architecture with a resolution of $\sim 7 \mathrm{~nm}$ by means of dual-axis electron tomography.

For the cis-Golgi assembly studies we have examined the structure of Golgi stacks in diverse cell types of Arabidopsis and alfalfa plants, and in the scale-forming alga Scherffelia dubia. All cell types exhibit the same structural intermediates, but due to the large size of the $S$. dubia Golgi stacks $(\sim 20$ cisternae, 1.5 micron diameter) that system has yielded the most detailed insights. Formation of a new cisterna starts with the formation of cis cisternal initials that arise from clusters of 3 to 5 tethered COPII-type vesicles that fuse after becoming linked to the C2 cis cisterna (Fig. 1). These initials grow by the fusion of individual COPII vesicles to localized sites giving rise to tubular outgrowths and eventually merge to form a mature new $\mathrm{C} 1$ cis cisterna. COPI-type vesicles are seen to bud off the developing $\mathrm{C} 1$ cisterna to recycle membrane molecules to the ER. All of these membrane assembly processes occur within a ribosome-excluding Golgi matrix.

The assembly of the cell plate during plant cell cytokinesis also occurs within a ribosome-excluding matrix, the cell plate assembly matrix (CPAM), within the phragmoplast (Fig. 2) [1, 2]. This process involves four phases: phragmoplast initials, solid phragmoplast, transitional and ring-shaped phragmoplast. Each of these phases is defined by the configuration of the phragmoplast microtubule (MT) array and the assembly state of the cell plate. One of the central challenges of cell plate formation is how to induce the fusing vesicles to form a planar membrane sheet and not a balloonlike structure. Upon entering the CPAM, the vesicles become connected through exocyst-type prefusion complexes, and the hourglass-shaped vesicle fusion intermediates become stabilized by dynamin spirals that expand to extend the neck to create dumbbell-shaped vesicles, which have a $50 \%$ reduction in volume, presumably through the loss of water. This dehydration causes gelling of the vesicle contents, which mechanically stabilizes the elongated vesicle form. Fusion of vesicles with these dumbbells produces bulbous strings that merge to form tubulovesicular networks (Fig. 3), which then mature into a new cell wall.

Analysis of the phragmoplast MT (+)-ends in dividing cells has led to the identification of 5 different end geometries: blunt (metastable), extended (growing), horned (disassembling), flared (beginning of disassembly), and hybrid extended /horned (rescue) (Fig. 4) [3]. Furthermore, of those MTs that 
end within the CPAM, $\sim 70 \%$ are blunt-ended, whereas of those that terminate outside the CPAM only $\sim 25 \%$ are blunt ended. One third of the blunt-ended MTs within the CPAM are connected to the cell plate via $30 \mathrm{~nm}$ linkers. These results indicate that the CPAM stabilizes MT $(+)$-ends in addition to providing a specialized environment for cell plate assembly.

Together, these findings demonstrate that the tomographic analysis of cryofixed / freeze-substituted cells can yield novel structural and mechanistic insights into cellular processes.

[1] M. S. Otegui et al., Plant Cell 13 (2001) 2033.

[2] J. M. Segui-Simarro et al., Plant Cell 16 (2004) 836.

[3] J. R. Austin et al., submitted

Supported by NIH grants GM61306 and GM59787 to LAS.
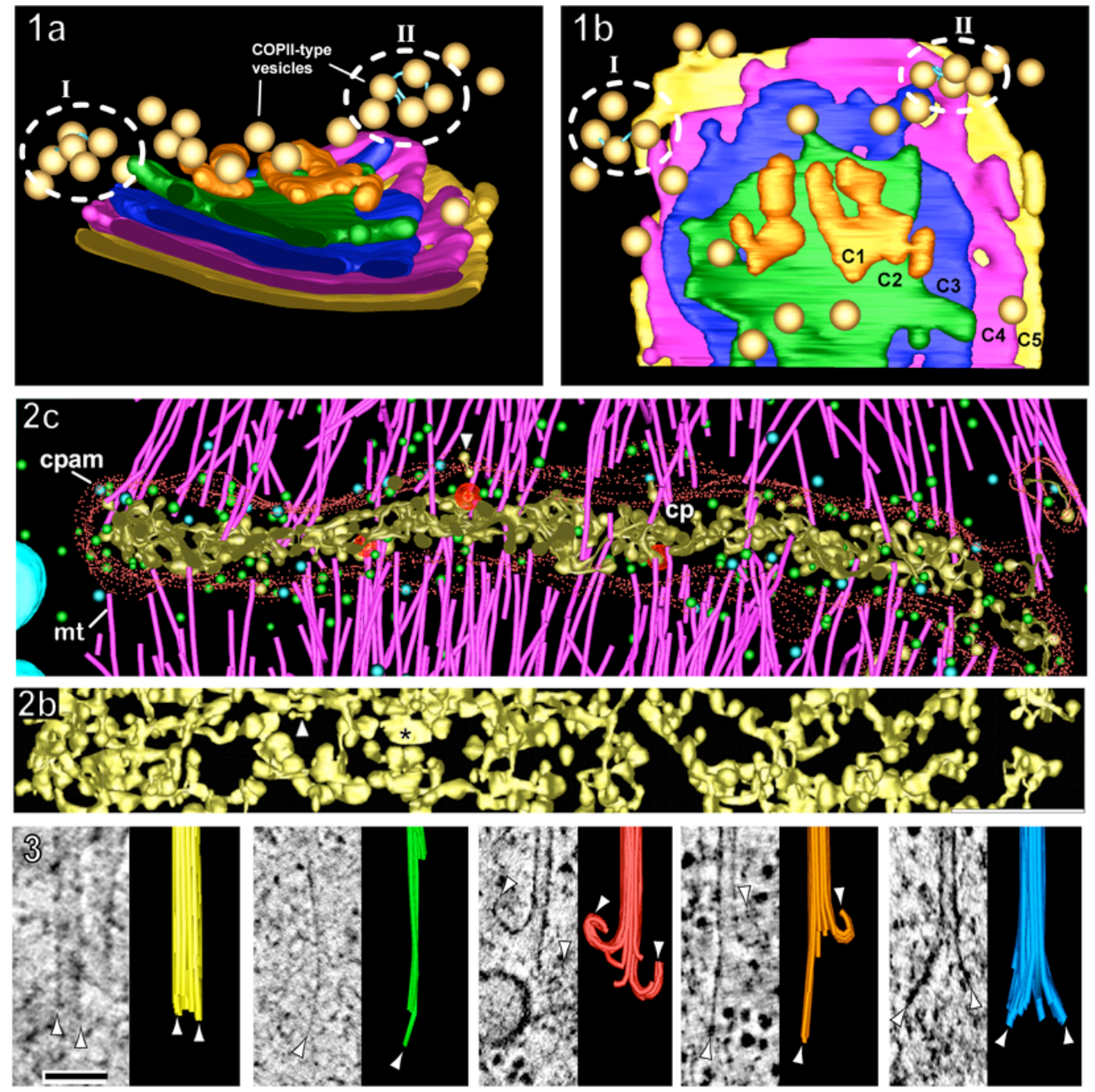

Figure 1: Cis-Golgi cisternae and associated COPII-type vesicles in the scale-forming alga Scherffelia dubia. (a) Side view with two clusters of tethered COPII vesicles. (b) Face-on view of the cisternae and vesicles seen in (a). Note the shapes of the assembling $\mathrm{C} 1$ cis-Golgi cisterna initials.

Figure 2: Central region of a reconstructed solid phragmoplast in a dividing Arabidopsis meristem cell. (a) Side view of a cell plate (cp); cell plate assembly matrix (cpam) that encompasses the cell plate; microtubule (mt). (b) Face on view of the cell plate shown in (a).

Figure 3: Tomographic images and 3D models of reconstructed phragmoplast microtubule (MT) (+)-ends. Yellow: blunt-ended, meta-stable MT; green: extended, growing MT; red: horned, disassembling MT; orange: hybrid (horned/extended), rescued MT; blue: flared, earliest stage of disassembling MT. 